\title{
Propriétés des lacticodéshydrogénases de Streptococcus thermophilus indépendantes du fructose 1,6-diphosphate
}

par

D. HEMME*, Michèle NARDI* et Danièle WAHL*

\section{INTRODUCTION}

Streptococcus thermophilus est une espèce aux caractères particuliers qui est utilisée en association avec Lactobacillus bulgaricus pour la fabrication du yaourt ou avec L. lactis ou L. helveticus pour celle des fromages à pâte cuite en France (Comté, Emmental, Beaufort), et également en Suisse. Les propriétés et les caractères taxonomiques des souches de cette espèce ont été récemment rassemblés (voir la revue Accolas et al. [2]). Le métabolisme particulier de cette espèce a été l'objet de plusieurs publications (voir in Hemme et al. [7]). Cette étude apporte certains éléments nécessaires à une meilleure connaissance des lacticodéshydrogénases (LDH) de S. thermophilus. En effet, à ce jour (voir la récente revue de Garvie [4]), seule une brève communication récente de Garvie (1978 [3]) et une plus ancienne, de Wolin (1964 [16]) apparaissent dans la littérature.

\section{MATERIELS ET METHODES}

\section{Souches}

Les souches de $S$. thermophilus étudiées appartenaient à la collection du Centre National de Recherches Zootechniques (CNRZ) de Jouy-en-Josas. L'origine des souches et leur mode de culture ont déjà été décrits [6]. Le milieu M 17 [14] a été utilisé.

\footnotetext{
* Institut National de la Recherche Agronomique, C.N.R.Z., Laboratoire de Microbiologie Laitière et de Génie Alimentaire - 78350 Jouy-en-Josas (France).
} 


\section{Préparation des extraits bruts solubles et partiellement purifiés}

Les extraits bruts solubles désignaient les surnageants obtenus après centrifugation $(13000 \mathrm{~g})$ des broyats cellulaires résultant d'un broyage mécanique [6].

Les LDH des souches 302 et 385 ont été partiellement purifiées. La fraction précipitable à 55 p. $100\left(\right.$ C.F. à $\left.0^{\circ} \mathrm{C}\right)$ de sulfate d'ammonium contenait la quasi-totalité des activités LDH. Les activités spécifiques augmentaient, par rapport à celles des extraits bruts, de 1 à 3,8 (souche 302 ) et de 1 à 3,4 (souche 385 ). Ces culots, repris dans le tampon TRIS $66 \mathrm{mM}$ pH 7,0 étaient indemnes de composants cellulaires non précipitables (substrats, coenzymes, ions) non liés aux enzymes.

\section{Détermination des protéines et de l'activité LDH}

La méthode de Lowry et al. [12] était utilisée pour le dosage des protéines. Les activités spécifiques étaient rapportées aux concentrations de protéines ainsi déterminées.

L'activité LDH était mesurée, soit en tampon phosphate de Na $50 \mathrm{mM}$ pH 7,5 contenant du pyruvate de $\mathrm{Na} 0,6 \mathrm{mM}$ et du coenzyme NADH $0,12 \mathrm{mM}$ (essais comparatifs avec les extraits bruts des dix souches), soit en tampon Tris- $\mathrm{HCl} 66 \mathrm{mM} \mathrm{pH} \mathrm{7,0} \mathrm{ou} \mathrm{acétate-acide}$ acétique $66 \mathrm{mM}$ pH 5,4 contenant du pyruvate $10 \mathrm{mM}$ et du NADH $0,15 \mathrm{mM}$ (essais avec les extraits purifiés). Les activités absolues étaient déterminées en comparant les diminutions de $\mathrm{D} . \mathrm{O} . / \mathrm{min}$ à $340 \mathrm{~nm}$ obtenues avec la LDH de Muscle de Lapin (Boehringer Mannheim) et avec les extraits de S. thermophilus. Dans nos conditions, on avait l'équivalence $0,1 \mathrm{U}$ D.O./min $=0,24 \mathrm{U} \mathrm{LDH} / \mathrm{ml}$. Pour pouvoir aisément déterminer la vitesse initiale, la dilution devait être telle (dilution 100 pour les extraits) que la variation de D.O. soit constante au moins $2 \mathrm{~min}$.

\section{Electrophorèse en gel de polyacrylamide}

L'étude de la mobilité électrophorétique des LDH a été effectuée en gel de polyacrylamide à 7 p. 100 (acrylamide 97 p. 100, bis acrylamide 3 p. 100) en tampon Tris- $\mathrm{HCl} 27 \mathrm{mM} \mathrm{pH} 8,6$ sous forme de plaque (épaisseur $2 \mathrm{~mm}$, longueur $180 \mathrm{~mm}$ ). Le tampon d'électrophorèse était du Tris-glycine $5 \mathrm{mM} \mathrm{pH} 8,6$. Les puits étaient chargés avec $0,05 \mathrm{ml}$ d'extrait (concentrations variant de 10 à $900 \mathrm{mg} / \mathrm{l}$ ). La migration était obtenue en $3 \mathrm{~h}$ à $400 \mathrm{~V}$ (32 mA, puissance max. $10 \mathrm{~W}$, redresseur LKB). La révélation était faite en 2 étapes. Les plaques étaient immergées 5 min dans un mélange contenant du NADH $(0,2 \mathrm{mM})$, du pyruvate $(10 \mathrm{mM})$, du phosphate $(20 \mathrm{mM})$ en tampon acide acétique-acétate

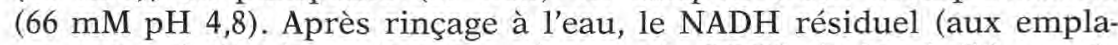
cements où il n'y avait pas présence de $\mathrm{LDH}$ ) était révélé par le mélange habituel de phénazine-méthosulfate et de MTT (bromure 
de 3 (4,5-diméthyl-thiazolyl-1-2) (0,63 $\mathrm{mM})$ 2,5-diphényl-tétrazolium $(6,5 \mu \mathrm{M})$.

\section{Propriétés des enzymes}

- Inactivation thermique : $1 \mathrm{ml}$ d'extrait était chauffé dans un tube de verre pendant des temps variables aux températures désirées, en l'absence ou en présence de divers composés (voir résultats). Après refroidissement immédiat dans la glace fondante, les activités résiduelles étaient mesurées.

- Stabilité à $4^{\circ} \mathrm{C}$ et à la congélation : les activités des extraits étaient mesurées aussitôt après broyage, puis après des temps variables de conservation à $4^{\circ} \mathrm{C}$ ou à $-20^{\circ} \mathrm{C}$. Dans ce dernier cas, la décongélation était faite dans un bain thermostaté à $30^{\circ} \mathrm{C}$.

- Effet du pH: les tampons acide acétique-acétate $66 \mathrm{mM}$ étaient utilisés entre $\mathrm{pH} \mathrm{4,6} \mathrm{et} \mathrm{pH}$ 6,2. Le tampon Tris- $\mathrm{HCl} 66 \mathrm{mM}$ permettait de connaître les activités à $\mathrm{pH} 7,0$.

- Constantes cinétiques et inhibition : les fractions précipitables par le sulfate d'ammonium à $55 \mathrm{p}, 100$ de saturation $\left(0^{\circ} \mathrm{C}\right)$ étaient utilisées pour déterminer l'affinité des LDH pour le pyruvate et le coenzyme NADH. Les activités étaient mesurées pour des concentrations variables de pyruvate $(0,1$ à $10 \mathrm{mM})$ en présence de NADH à concentration saturante $(0,2 \mathrm{mM})$ et pour des concentrations variables de NADH ( $1 \mu \mathrm{M}$ à $1 \mathrm{mM}$ ) en présence de pyruvate à concentration saturante $(10 \mathrm{mM})$.

\section{RESULTATS}

\section{Activités spécifiques}

Les extraits de toutes les souches sont capables d'oxyder le $\mathrm{L}(+)$-lactate, mais non le $\mathrm{D}(-)$-lactate, en pyruvate. Leurs enzymes sont donc uniquement de type L-LDH. Cela concorde avec l'isomère lactique produit dans le milieu de culture. Les activités mesurées dans le sens de la réduction du pyruvate sont environ trente fois plus fortes que celles mesurées dans le sens de l'oxydation du L (+)-lactate.

Les activités spécifiques LDH des extraits bruts de dix souches, mesurées dans le sens de la réduction du pyruvate, varient selon les souches (tab. 1). On peut distinguer deux groupes dénommés I et II dont les activités sont sensiblement dans un rapport de 2 à 1 . Les activités spécifiques ainsi mesurées sont sans rapport avec la concentration en acide lactique du milieu.

\section{Stabilité comparée des extraits de dix souches}

L'activité résiduelle LDH des extraits des souches 21 et 302 après 1 an de conservation à $-20^{\circ} \mathrm{C}$ est notablement plus élevée que 
TABLEAU 1

Culture des souches de Streptococcus thermophilus en milieu M 17, teneur en protéines des extraits et activités spécifiques des lacticodéshydrogénases

\begin{tabular}{|c|c|c|c|c|c|c|c|c|c|c|}
\hline \multirow{2}{*}{$\begin{array}{l}\text { Groupes } \\
\text { souches }\end{array}$} & \multicolumn{3}{|c|}{ Groupe I } & \multicolumn{7}{|c|}{ Groupe II } \\
\hline & 160 & 21 & 391 & 445 & 307 & 7 & 302 & 368 & 385 & 308 \\
\hline $\begin{array}{l}\text { Activités spécifiques : } \\
\text { en } \mathrm{U} / \mathrm{mg} \\
\text { en p. } 100\end{array}$ & $\begin{array}{r}1,8 \\
100\end{array}$ & 76 & 76 & 55 & 54 & 46 & 44 & 43 & 42 & 32 \\
\hline $\begin{array}{l}\text { Concentration en acide lac- } \\
\text { tique des cultures }(\mathrm{g} / \mathrm{l})^{*}\end{array}$ & 3,97 & 4,02 & 4,54 & 3,90 & 4,09 & 4,16 & 3,55 & 3,55 & 3,90 & 4,44 \\
\hline $\begin{array}{l}\text { Concentration en protéines } \\
\text { des extraits }(\mathrm{g} / \mathrm{l})\end{array}$ & 0,82 & 1,60 & 2,10 & 1,96 & 1,20 & 2,07 & 1,46 & 1,83 & 1,10 & 2,60 \\
\hline
\end{tabular}

celle des quatre autres souches testées (tab. 2). Les LDH sont par ailleurs stables à $4^{\circ} \mathrm{C}$ pendant plusieurs jours, à condition que les concentrations en protéines soient $>1 \mathrm{~g} / 1$.

\section{Inactivation thermique}

Les LDH des souches 445, 385 et 302 apparaissent plus thermostables que celles des autres souches (fig. 1), ces trois souches appar-

\section{TABLEAU 2}

Stabilité des lacticodéshydrogénases contenues dans les extraits bruts solubles de Streptococcus thermophilus

\begin{tabular}{|c|c|c|c|c|c|c|c|c|c|c|}
\hline \multirow{2}{*}{$\begin{array}{l}\text { Groupes } \\
\text { souches }\end{array}$} & \multicolumn{3}{|c|}{ Groupe I } & \multicolumn{7}{|c|}{ Groupe II } \\
\hline & 160 & 21 & 391 & 445 & 307 & 7 & 302 & 368 & 385 & 308 \\
\hline $\begin{array}{l}\text { Activités résiduelles (A.R.) } \\
\text { après } 1 \text { an de congélation } \\
\text { à }-20^{\circ} \mathrm{C} \text { (en p. } 100 \text { de l'ac- } \\
\text { tivité initiale) }\end{array}$ & 3 & 26 & $\mathrm{ND}^{1}$ & 9 & 5 & ND & 19 & ND & 4 & ND \\
\hline $\begin{array}{l}\text { A.R. après } 5 \mathrm{~min} \text { de chauf- } \\
\text { fage à } 59^{\circ} \mathrm{C}\end{array}$ & 4 & 5 & 5 & 18 & 5 & 4 & 18 & 13 & 5 & 6 \\
\hline
\end{tabular}

1. ND : non déterminé. 


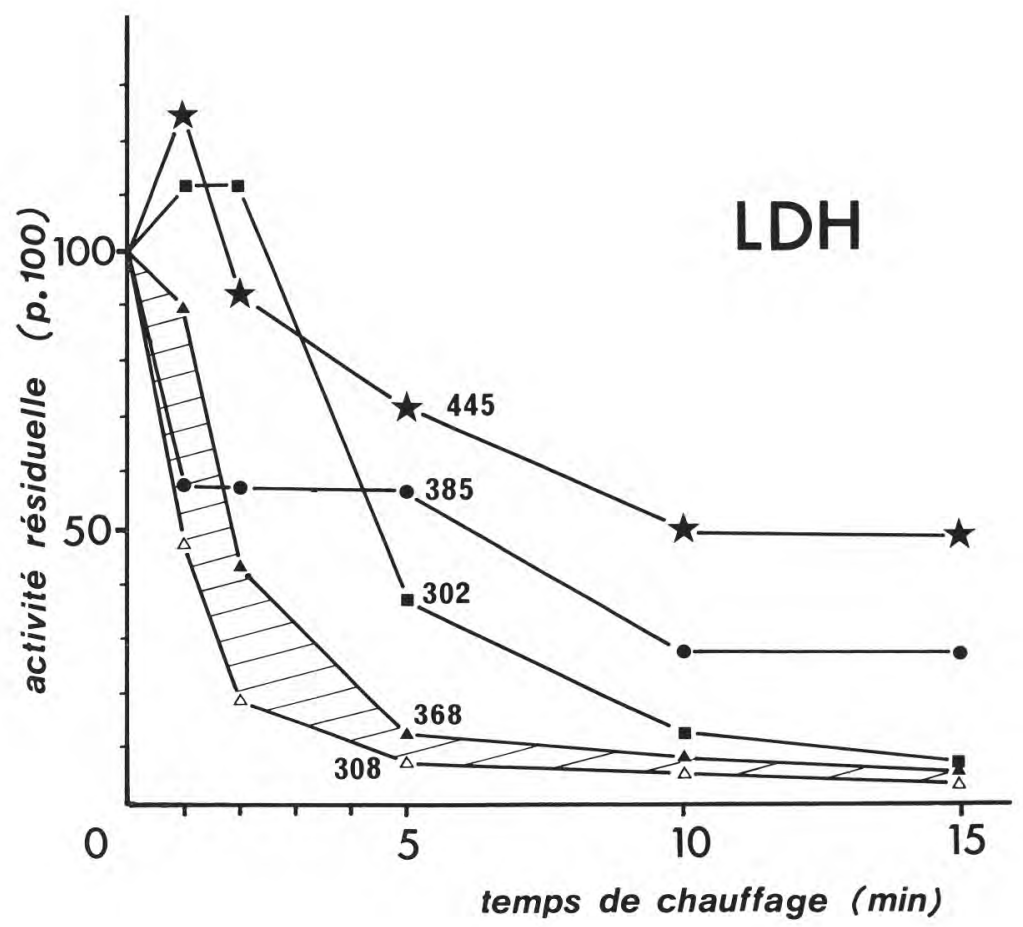

fig. 1

Effet du chauffage des extraits bruts solubles de Streptococcus thermophilus sur les lacticodéshydrogénases

Les extraits bruts solubles étaient chauffés des temps variables à $55^{\circ} \mathrm{C}$, refroidis dans la glace, et l'activité résiduelle était mesurée à $30^{\circ} \mathrm{C}$. Les courbes obtenues pour les cinq souches non représentées se situent dans la partie hachurée.

tenant au groupe II. Toutefois, la LDH de la souche 385 n'est pas activée par des temps de chauffage courts comme le sont les LDH des souches 302 et 445 . Cette différence de comportement est confirmée lors du chauffage à $59^{\circ} \mathrm{C}$ (tab. 2). La résistance au chauffage n'est apparemment pas en relation avec la résistance à la congélation.

Les activités résiduelles des extraits chauffés en présence des substrats ou coenzyme sont égales ou supérieures à celles des témoins (tab. 3). Le pyruvate protège les $\mathrm{LDH}$ de trois des quatre souches, celle de la souche 302 ne l'étant pas. Le D-lactate, bien que n'étant pas substrat, protège aussi bien les LDH que le L-lactate pour trois souches sur les quatre. 


\section{TABLEAU 3}

Protection des LDH de Streptococcus thermophilus vis-à-vis du chauffage par les substrats, un analogue de substrat et un coenzyme

\begin{tabular}{|c|c|c|c|c|c|c|}
\hline \multirow{2}{*}{\multicolumn{2}{|c|}{$\begin{array}{l}\text { Groupes } \\
\text { souches }\end{array}$}} & \multirow{3}{*}{$\begin{array}{c}\mathrm{I} \\
160 \\
43^{\circ} \mathrm{C}\end{array}$} & \multicolumn{4}{|c|}{ II } \\
\hline & & & 445 & 307 & 302 & 385 \\
\hline Température de chauffage & $\min$ à & & $57^{\circ} \mathrm{C}$ & $43^{\circ} \mathrm{C}$ & $50^{\circ} \mathrm{C}$ & $57^{\circ} \mathrm{C}$ \\
\hline $\begin{array}{l}\text { Activité résiduelle après chauf- } \\
\text { fage en absence (témoin) ou } \\
\text { en présence des substrats ci- } \\
\text { contre ( } 2 \mathrm{mM})\end{array}$ & $\begin{array}{l}\text { témoin } \\
\text { NADH } \\
\text { NAD } \\
\text { pyruvate } \\
\text { L-lactate } \\
\text { D-lactate }\end{array}$ & $\begin{array}{l}80 \\
80 \\
80 \\
90 \\
90 \\
85\end{array}$ & $\begin{array}{l}60 \\
60 \\
70 \\
80 \\
90 \\
90\end{array}$ & $\begin{array}{r}70 \\
70 \\
70 \\
100 \\
90 \\
90\end{array}$ & $\begin{array}{l}80 \\
80 \\
80 \\
70 \\
90 \\
90\end{array}$ & $\begin{array}{r}80 \\
80 \\
80 \\
115 \\
90 \\
70\end{array}$ \\
\hline
\end{tabular}

\section{Fractionnement et propriétés de 2 LDH chez les souches 302 et 385}

Tant pour les souches 302 que 385, la fraction précipitable par le sulfate d'ammonium à $55 \mathrm{p} .100$ de saturation (à $0^{\circ} \mathrm{C}$ ) renferme la majeure partie de l'activité LDH. Cette technique permet d'éliminer les petites molécules non précipitables contenues dans les extraits bruts et d'augmenter les activités spécifiques LDH (tab. 4).

\section{TABLEAU 4}

Activités spécifiques des extraits de Streptococcus thermophilus

\begin{tabular}{|c|c|c|c|c|c|}
\hline Souche & Extrait & $\begin{array}{l}\text { Concentration } \\
\text { en protéines } \\
(\mu \mathrm{g} / \mathrm{ml})\end{array}$ & $\begin{array}{l}\text { Activité } \\
\text { (U/ml) }\end{array}$ & $\begin{array}{l}\text { Activité } \\
(\mathrm{U} / \mathrm{mg})\end{array}$ & $\begin{array}{l}\text { spécifique } \\
\text { (en p. 100) }\end{array}$ \\
\hline 302 & $\begin{array}{l}\text { Extrait brut (EB) } \\
\text { Culot* } 55 \mathrm{p} .100 \\
\text { Surnageant } 55 \mathrm{p} .100\end{array}$ & $\begin{array}{r}660 \\
150 \\
9\end{array}$ & $\begin{array}{c}21,6 \\
19,8 \\
1,86\end{array}$ & $\begin{array}{l}32,7 \\
132 \\
207\end{array}$ & $\begin{array}{l}100 \\
404 \\
633\end{array}$ \\
\hline 385 & $\begin{array}{l}\text { Extrait brut (EB) } \\
\text { Culot* } 55 \text { p. } 100 \\
\text { Surnageant } 55 \text { p. } 100\end{array}$ & $\begin{array}{r}910 \\
295 \\
8\end{array}$ & $\begin{array}{r}34,2 \\
30,6 \\
2,7\end{array}$ & $\begin{array}{l}37,6 \\
103,7 \\
337\end{array}$ & $\begin{array}{l}100 \\
276 \\
896\end{array}$ \\
\hline
\end{tabular}

* Obtenus par addition de sulfate d'ammonium à 55 p. 100 de saturation, à $0^{\circ} \mathrm{C}$ et remis en solution dans un volume de tampon égal au volume de l'extrait brut. 


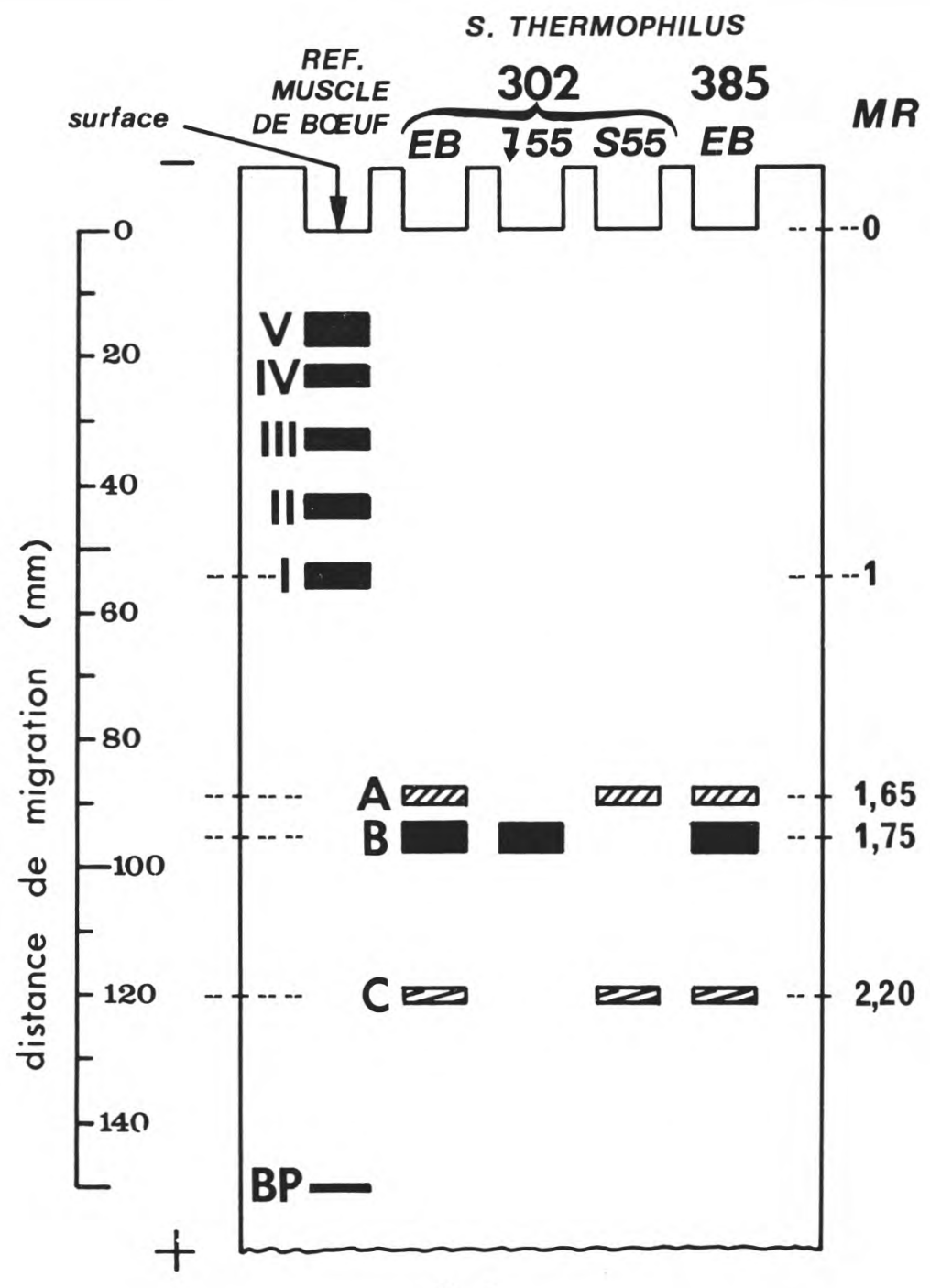

fig. 2

Mobilités électrophorétiques des lacticodéshydrogénases de Streptococcus thermophilus

I à V : isoenzymes de l'extrait de muscle de bœuf utilisé (l'isoenzyme I migre de façon analogue à l'isoenzyme I du muscle de lapin. Il sert de référence).

BP : bleu de bromophénol, indiquant le front de migration.

A, B et C : LDH de $S$. thermophilus.

MR : mobilités relatives (isoenzyme $\mathrm{I}=1$ ).

Dépôts : $50 \mu \mathrm{l}$ d'extraits contenant $30 \mu \mathrm{g}$ (EB 302), $45 \mu \mathrm{g}$ (EB 385), $7 \mu \mathrm{g}$ ( 755 ) ou $0,5 \mu \mathrm{g}$ (S 55) de protéines. Migration pendant $3 \mathrm{~h}$, tension $400 \mathrm{~V}$; gel de polyacrylamide de $7 \mathrm{p} .100, \mathrm{pH} 8,6$. Révélation en tampon acide acétique-acétate $\mathrm{pH} 4,8$, pyruvate $10 \mathrm{mM}$, NADH 0,2 mM, $\mathrm{PO}_{4} 20 \mathrm{mM}$; lavage ; coloration du NADH résiduel par un mélange de PMS $(6,5 \mu \mathrm{M})$ et de MTT $(0,63 \mathrm{mM})$. 
L'électrophorèse en gel de polyacrylamide montre (fig. 2) que la fraction précipitable à 55 p. 100 contient celle des deux enzymes LDH présentes dans l'extrait brut qui est la plus active (B : mobilité relative par rapport à l'isoenzyme I, 1,75). L'autre enzyme LDH, présente dans le surnageant, a une mobilité électrophorétique plus faible $(A, 1,65)$. L'activité $C$ (mobilité relative 2,20) également détectée dans le surnageant, est très faible et pourrait correspondre à la faible activité NADH-oxydase, détectée lors des dosages, surtout pour la souche $302(0,4 \mathrm{U} / \mathrm{ml})$. Les extraits de la souche 385 conduisent aux mêmes résultats, tant pour l'extrait brut (fig. 2) que pour les fractions précipitable et non précipitable (non représentées).

\section{Propriétés des LDH de type B (précipitables par le sulfate d'ammo- nium à 55 p. 100) de $S$, thermophilus CNRZ 302 et 385}

- Conditions de mesure des activités.

Le tableau 5 montre que les fractions précipitables à 55 p. 100 doivent être diluées environ cent fois pour que les vitesses initiales

\section{TABLEAU 5}

Effet de la concentration d'enzyme sur la cinétique de la réaction de réduction du pyruvate chez Streptococcus thermophilus

\begin{tabular}{c|c|c}
\hline Dilution de l'extrait & Cinétique linéaire (temps) & Variation de DO/min \\
\cline { 2 - 3 } 10 & $15 \mathrm{~s}$ & \\
\hline 20 & $30 \mathrm{~s}$ & 0,650 \\
30 & $70 \mathrm{~s}$ & 0,310 \\
40 & 1 min $30 \mathrm{~s}$ & 0,206 \\
50 & 2 min & 0,139 \\
60 & $3 \mathrm{~min}$ & 0,116 \\
80 & $>5$ min & 0,084 \\
100 & & 0,061 \\
& & 0,049 \\
\hline
\end{tabular}

Extrait : fraction précipitable par le sulfate d'ammonium à 55 p. 100 de saturation, souche 302 .

puissent être mesurées avec précision. Dans les conditions standard utilisées (NADH 0,15 mM, pyruvate $10 \mathrm{mM}$ ), la concentration en protéines est alors, pour les dilutions au 1/100, de $6 \mathrm{mg} / \mathrm{l}$.

- Effet du $p H$.

Les LDH des deux souches 302 et 385 possèdent des activités mesurées dans le sens de la réduction du pyruvate, qui sont voisines, 


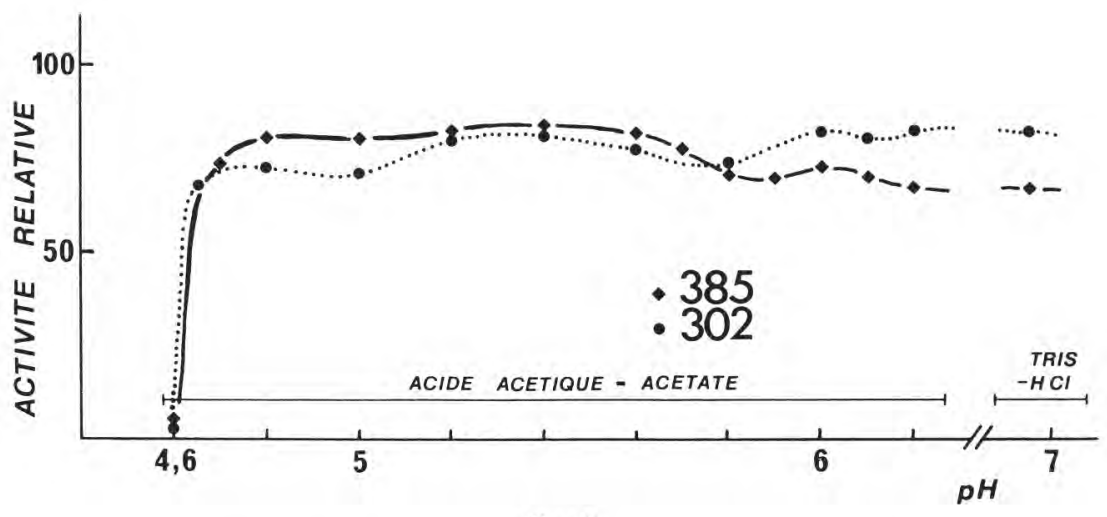

fig. 3

Effet du pH sur l'activité des lacticodéshydrogénases de Streptococcus thermophilus 302 et 385

Les activités étaient mesurées par la variation de $\mathrm{DO} / \mathrm{min}$ selon la méthode cinétique (voir matériels et méthodes). Pyruvate $10 \mathrm{mM}$, NADH $0,2 \mathrm{mM}$, tampons $66 \mathrm{mM}$, fractions précipitables par le sulfate d'ammonium à $55 \mathrm{p} .100,250 \mathrm{ng} / \mathrm{ml}$.

entre $\mathrm{pH} 4,7$ et $\mathrm{pH}$ 7,0 (fig. 3). Les profils sont voisins avec une chute très brusque de l'activité entre $\mathrm{pH} 4,65$ et $\mathrm{pH} \mathrm{4,60.}$

\section{Courbes de saturation par le coenzyme NADH}

Les concentrations en $\mathrm{NADH}$ saturantes pour les $\mathrm{LDH}$ des souches 302 et 385 (fig. 4) sont différentes ( $K_{x}$ respectives 86 et $33 \mu \mathrm{M}$ ). Il y a inhibition par excès de NADH pour les LDH des deux souches pour des concentrations $>0,15 \mathrm{mM}$ (souche 302 ) ou $>0,3 \mathrm{mM}$ (souche 385). La V max de la souche 385 est par ailleurs plus faible $(80 \mathrm{U} / \mathrm{ml})$ que celle de la souche $302(124 \mathrm{U} / \mathrm{ml})$. La concentration en NADH $0,2 \mathrm{mM}$ a été retenue pour les conditions standard de mesure de l'activité LDH (substrats en concentrations saturantes).

\section{Inhibitions par divers analogues}

Parmi les composés testés (tab. 6), ce sont surtout l'ATP, l'ADP, le NAD et l'oxamate qui sont inhibiteurs des $\mathrm{LDH}$, et ceci pour des concentrations $1 \mathrm{mM}$. Des inhibitions moins fortes ont lieu avec d'autres composés, mais en concentration égale à celle du pyruvate $(10 \mathrm{mM})$. Les inhibitions sont généralement plus fortes à $\mathrm{pH} 5,4$ qu'à $\mathrm{pH} \mathrm{7,0.} \mathrm{Cela} \mathrm{est} \mathrm{en} \mathrm{particulier} \mathrm{le} \mathrm{cas} \mathrm{pour} \mathrm{le} \mathrm{NAD.} \mathrm{On} \mathrm{peut} \mathrm{noter} \mathrm{le}$ faible pouvoir inhibiteur du L-lactate. 


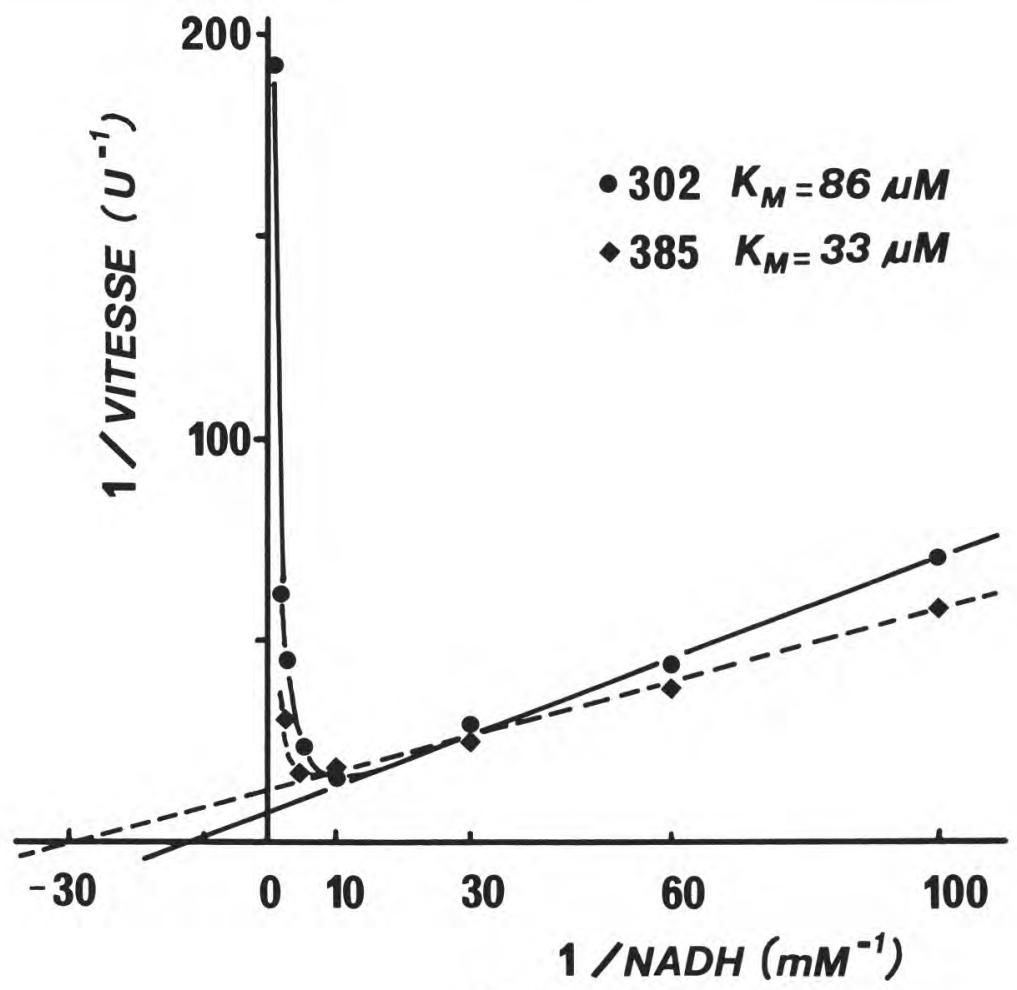

fig. 4

Activité des lacticodéshydrogénases de Streptococcus thermophilus 302 et 385 en fonction de la concentration de coenzyme NADH

Représentation en coordonnées inverses de Lineweaver et Burk [11].

Les mesures étaient effectuées par la méthode cinétique $(\Delta \mathrm{DO} / \mathrm{min}$; voir matériels et méthodes).

Tampon TRIS- $\mathrm{HCl}, 66 \mathrm{mM}, \mathrm{pH} 7,0$, pyruvate $10 \mathrm{mM}$, FDP $0,5 \mathrm{mM}$, fractions précipitables par le sulfate d'ammonium à $55 \mathrm{p} .100,250 \mathrm{ng} / \mathrm{ml}$.

\section{Courbes de saturation par le pyruvate en l'absence et en présence de certains effecteurs}

La figure 5 donne, en coordonnées inverses, les courbes obtenues. Le fructose-1-6-diphosphate (FDP, 0,5 mM) a peu d'influence sur l'activité LDH. Il est légèrement inhibiteur pour la souche 385 et par contre augmente l'affinité de la LDH de la souche 302. Des concentrations de FDP plus fortes $(0,5$ à $10 \mathrm{mM})$ n'ont pas plus d'effet. Sans FDP, les courbes donnant $\mathrm{V}$ en fonction de la concentration en pyruvate sont hyperboliques et non pas sigmoïdes comme cela a été décrit pour d'autres LDH [9]. 


\section{TABLEAU 6}

Action de divers composés sur l'activité lacticodéshydrogénasique de Streptococcus thermophilus CNRZ 302

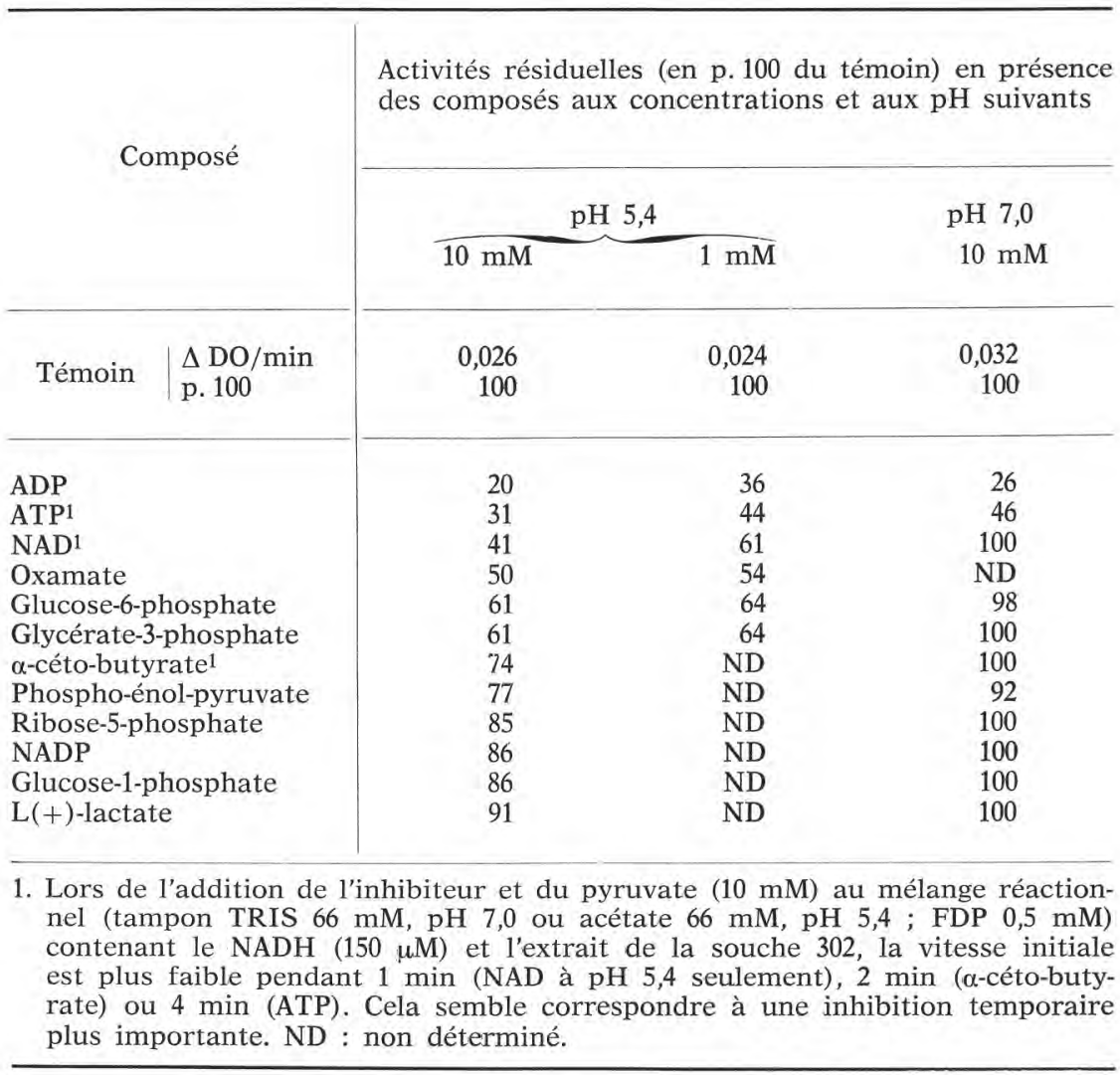

De même, tant à $\mathrm{pH} 5,4$ qu'à $\mathrm{pH} 7,0$, les ions $\mathrm{Mn}^{++}$, en présence ou en absence de FDP, sont faiblement inhibiteurs (7 p. 100) pour la LDH de la souche 302 (dès la concentration $0,33 \mathrm{mM}$ ) et n'ont aucun effet sur la LDH de la souche 385. Par contre, les ions phosphate (20 mM) sont activateurs (fig. 5), avec ou sans FDP, permettant surtout une augmentation de la vitesse de réaction ainsi qu'une légère augmentation de l'affinité des LDH pour le pyruvate.

L' $\alpha$-céto-butyrate $(10 \mathrm{mM})$ donne la même inhibition, avec ou sans FDP $(0,5 \mathrm{mM})$, diminuant la vitesse de réaction. Le NAD $(5 \mathrm{mM})$ agit d'une façon analogue. Enfin, tant en présence de ces divers effecteurs qu'en leur absence, on note pour les deux souches une inhibition par excès de pyruvate pour les concentrations $>10 \mathrm{mM}$ pour la 


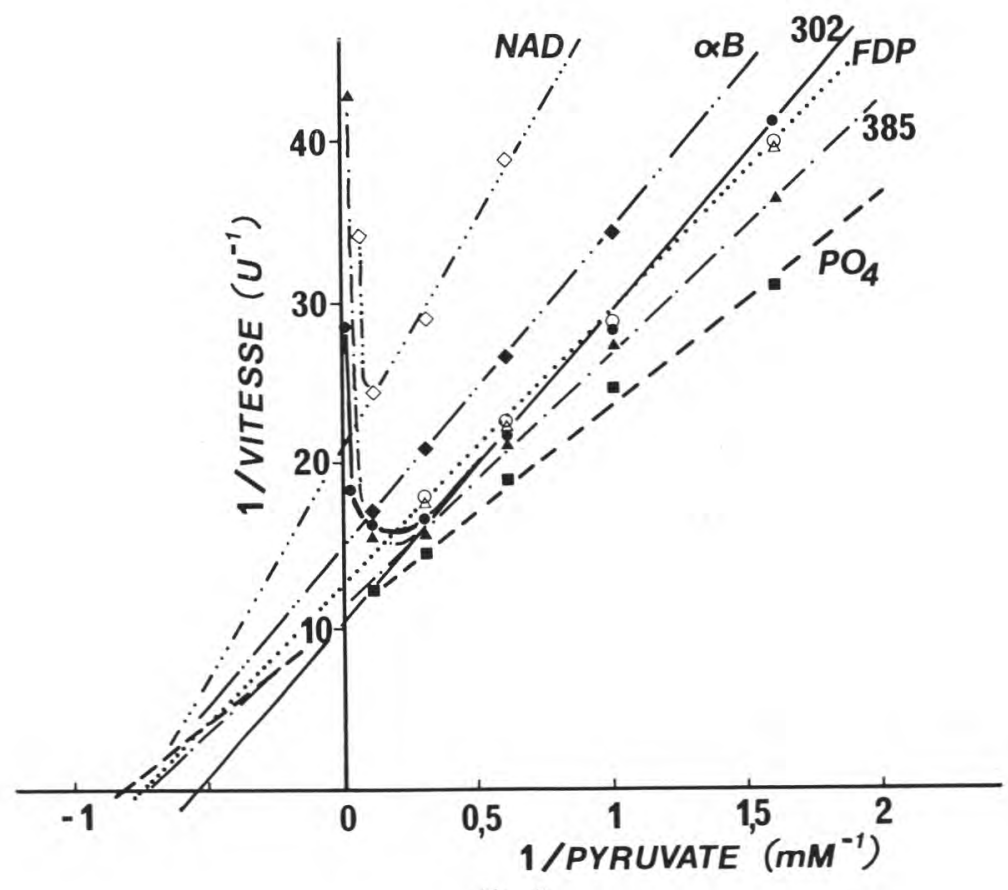

fig. 5

Activité des lacticodéshydrogénases de Streptococcus thermophilus 302 et 385 en fonction de la concentration de pyruvate.

Effet de l'addition de NAD, d' $\alpha$-céto-butyrate, de fructose-1-6-diphosphate ou de phosphate au mélange réactionnel

Représentation en coordonnées inverses selon Lineweaver et Burk [11].

Les mesures étaient effectuées par la méthode cinétique $(\Delta \mathrm{DO} / \mathrm{min}$; voir matériels et méthodes). Souche 302 testée sans addition (302) ou avec addition de FDP $0,5 \mathrm{mM}$ (FDP), de NAD $5 \mathrm{mM}$ (NAD) ou de $\mathrm{PO}_{4} 20 \mathrm{mM}\left(\mathrm{PO}_{4}\right)$; souche 385 testée sans addition (385) ou avec addition de FDP $0,5 \mathrm{mM}$ (courbe confondue avec celle obtenue pour la souche 302 avec addition de FDP) ou d'a-céto-butyrate $10 \mathrm{mM}(\alpha \mathrm{B})$. Tampon acide acétique-acétate $66 \mathrm{mM} \mathrm{pH} \mathrm{5,4,} \mathrm{NADH} \mathrm{0,2} \mathrm{mM,}$ fractions précipitables par le sulfate d'ammonium à 55 p. 100, $250 \mathrm{ng} / \mathrm{ml}$.

souche $385\left(\mathrm{~K}_{\mathrm{M}}=1,4 \mathrm{mM}\right)$ et $>50 \mathrm{mM}$ pour la souche $302\left(\mathrm{~K}_{\mathrm{M}}=1,8\right.$ $\mathrm{mM})$. De ce fait, la concentration $10 \mathrm{mM}$ était la concentration saturante utilisée pour les mesures d'activités standard.

\section{Propriété de la LDH de type A (non précipitable par le sulfate d'ammonium à 55 p. 100) de $S$. thermophilus CNRZ 302}

L'activité LDH de la fraction non précipitable par le sulfate d'ammonium à 55 p. 100, résultant de la présence de la $\mathrm{LDH}$ de type A 
est mieux protégée des effets du chauffage par le FDP que celle de type B. De plus, l'ion $\mathrm{Mn}^{++}(33 \mathrm{mM}$ ) protège cette $\mathrm{LDH}$ de type A des effets du chauffage alors qu'il ne protège pas, voire même renforce ce même effet sur la LDH de type B.

L'affinité de la LDH de type A pour le pyruvate est plus faible que celle de la $\mathrm{LDH}$ de type $\mathrm{B}\left(\mathrm{K}_{\mathrm{M}}=1,1 \mathrm{mM}\right.$ contre $\left.1,8 \mathrm{mM}\right)$ en l'absence de FDP. Ce dernier active la LDH de type A de 24 p. 100 (mesure d'activité dans les conditions standard).

\section{DISCUSSION}

Pour mesurer avec suffisamment de précision les vitesses des réactions (vitesse d'oxydation du NADH en NAD), nous avons utilisé des extraits, bruts ou partiellement purifiés, qui soient suffisamment dilués. La pente à l'origine est alors confondue avec la courbe enregistrée, ce qui n'est pas le cas lorsque les variations de D.O. par unité de temps sont trop fortes [3].

La LDH de $S$. thermophilus est trouvée stable à $4^{\circ} \mathrm{C}$, ce qui confirme les observations de Garvie [3]. La courbe biphasique obtenue lors du chauffage de l'extrait de la souche 385 pourrait être le reflet de l'existence de deux isoenzymes. Toutefois l'activité totale LDH est due pour la plus grande part à la LDH de type électrophorétique $B$. On pourrait dès lors penser que cette courbe bi-phasique puisse révéler la dénaturation d'une enzyme oligomérique en sousunités moins actives. Toutefois, il est probable que la LDH de type B diffère des $\mathrm{LDH}$ isolées et purifiées d'autres bactéries lactiques [5, 13]. La LDH de type B de S. thermophilus n'est pas activée par le FDP [3] comme le sont les enzymes multimériques de $L$. casei $[5,9]$ ou des streptocoques du groupe $\mathrm{N}[10,15]$. De plus, les courbes donnant l'activité en fonction de la concentration en pyruvate n'ont pas l'allure sigmoïde trouvée pour les LDH de L. casei ou L. curvatus $[5,9]$ et qui deviennent hyperboliques en présence de FDP. Il pourrait donc ne pas y avoir d'effets coopératifs dans le cas de la LDH de $S$. thermophilus. Cela expliquerait alors l'inhibition par excès de $\mathrm{NADH}$ et par excès de pyruvate trouvée dans cette étude pour les LDH de type B et qui ne se manifeste pas chez les L-LDH déjà décrites $[5,9]$. De plus, contrairement aux L-LDH de $L$. casei et $L$. curvatus, les LDH de type B sont activées par les ions phosphate.

Bien que le FDP n'active pas les $\mathrm{LDH}$ de $S$. thermophilus, cette molécule est très certainement capable de se lier aux enzymes puisqu'elle les protège des effets du chauffage (tant la LDH de type B que la LDH de type A). Cette possibilité de liaison avec les LDH est confirmée par les essais montrant que l'inhibition par les ions $\mathbf{M n}^{++}$ est plus faible en présence qu'en absence de FDP. L'effet du FDP est très certainement dû au groupe phosphate. Les ions $\mathrm{PO}_{4}$ sont 
activateurs des LDH de type B. Ils permettent une augmentation de la vitesse de réaction sans pour autant changer l'affinité des enzymes pour le pyruvate. On peut donc penser qu'ils se lient à l'enzyme au niveau d'un site effecteur. C'est à ce même site que doit se lier le FDP et d'autres composés phosphorylés. Par contre les composés qui sont fortement inhibiteurs ont une structure analogue au NADH (ADP, ATP) ou au pyruvate (alpha-cétobutyrate, oxamate) et entrent en compétition avec le NADH et le pyruvate [5].

Le $\mathrm{L}(+)$-lactate a été trouvé très faiblement inhibiteur, ce qui tendrait à indiquer qu'il se fixe après le NADH sur les LDH de $S$. thermophilus $[10,15]$ ou bien qu'il a peu d'affinité pour les LDH [5] comme en témoigne l'activité beaucoup plus faible détectée dans le sens de l'oxydation de ce composé en présence de NAD. Ce manque d'affinité du lactate pour les LDH n'est pas sans rapport avec les conditions dans lesquelles se trouvent les LDH dans le cytoplasme cellulaire, notamment lorsque les bactéries sont en fin de croissance, dans un milieu contenant une concentration élevée de lactate. Si ce n'est par sa concentration, le lactate peut en effet entraîner une chute du $\mathrm{pH}$ intracellulaire. La chute brutale de l'activité des LDH de type B observée dans cette étude $(\mathrm{pH} \mathrm{4,65)}$ impose en effet que le $\mathrm{pH}$ intracellulaire se maintienne au-dessus de cette valeur tant que la croissance et la production d'acide ont lieu. Or, bien que la souche 385 soit capable de se développer et de produire l'acide lactique jusqu'à un $\mathrm{pH}$ plus bas que la souche $302(\mathrm{pH} 4,5$ contre $\mathrm{pH} 5,0$ [1]), les LDH des deux souches ont les mêmes propriétés, notamment en fonction du $\mathrm{pH}$. Nous confirmons ainsi, au niveau des LDH, l'hypothèse faite précédemment [8] impliquant une meilleure régulation du $\mathrm{pH}$ interne dans le cas de la souche 385 , que celle de la souche 302 . On peut penser que la souche 385 possède des systèmes plus efficaces d'excrétion du lactate vers le milieu extérieur, de telle sorte que le $\mathrm{pH}$ interne des cellules de cette souche reste plus élevé que celui de la souche 302 lorsque le $\mathrm{pH}$ du milieu de culture descend.

Cette étude, utilisant des souches de $S$. thermophilus qui ont donné de bons résultats en fabrication fromagère [1] et qui peuvent être prises comme des références de l'espèce, confirme la particularité de cette espèce, bien différente des streptocoques du groupe N. Elle confirme les éléments partiels fournis par Garvie [3]. De ce fait, on peut plutôt penser, contrairement à l'opinion émise par cet auteur [3], que $S$. thermophilus est une espèce homogène en ce qui concerne les LDH. La souche de $S$. thermophilus (108 de la Collection de 1'Université de l'Illinois) utilisée par Wolin [16] et qui possédait une activité LDH activée par le FDP était très certainement une souche qui n'appartient pas vraiment à l'espèce $S$, thermophilus, ou bien qui représente une exception. L'espèce $S$. thermophilus ne posséderait donc pas, comme c'est le cas pour $S$. mutans (17) des souches dont les LDH sont activées par le FDP et des souches dont les LDH ne sont pas activées par ce composé. 


\section{Remerciements}

Nous remercions J.P. Accolas pour l'aide qu'il nous a apportée pour la culture des souches et lors de la rédaction du manuscrit, Marie-France Dorbe pour certains dosages, $\mathrm{J}$. Bousset pour la réalisation des électrophorèses, Nicole Pamboukdjian et Elisabeth Hemme pour les traductions anglaise et allemande.

\section{Rés u mé}

Ce travail était consacré aux propriétés des lacticodéshydrogénases (LDH) de Streptococcus thermophilus. Pour effectuer cette étude, des extraits bruts solubles de dix souches et des extraits partiellement purifiés de deux de ces dix souches ont été utilisés.

La stabilité au froid et à la congélation ainsi que la résistance thermique variaient selon les souches. Les LDH étaient activées par l'ion phosphate mais ne l'étaient pas par le FDP ; ce composé est inhibiteur à forte concentration $(>1 \mathrm{mM})$. L'ion $\mathrm{Mn}^{++}$inhibait légèrement les LDH. L'ADP, l'ATP, le 6-P-gluconate, l'oxamate et le NAD étaient les inhibiteurs les plus forts de l'activité LDH mesurée dans le sens de la réduction du pyruvate. Certaines de ces inhibitions variaient avec le $\mathrm{pH}$. L'activité dans le sens de l'oxydation du $\mathrm{L}(+)$-lactate était trente fois plus faible que l'activité de réduction du pyruvate et elle exigeait de très fortes concentrations en lactate $(>200 \mathrm{mM})$. Un excès de NADH $(0,2 \mathrm{mM}$ pour la souche $302,0,33 \mathrm{mM}$ pour la souche 385 ) inhibait l'activité LDH. Le pyruvate était également inhibiteur $(>10 \mathrm{mM})$ et son effet était différent selon que le FDP était présent $(0,5 \mathrm{mM})$ ou non. Les $\mathrm{K}_{\mathrm{u}}$ et les $\mathrm{V}_{\max }$ ont été mesurées. L'activité LDH demeurait constante dans une large zone de $\mathrm{pH}(4,7$ à 7,5$)$. Toutefois, elle chutait très rapidement aux $\mathrm{pH}<4,7$, devenant quasi nulle à $\mathrm{pH} 4,6$. Les mobilités électrophorétiques des LDH de $S$. thermophilus étaient comparées à celles, bien connues, des isoenzymes du muscle bovin. Deux LDH de mobilités électrophorétiques différentes étaient détectées : l'une de type A soluble à une concentration de 55 p. 100 de sulfate d'ammonium, l'autre de type B (la plus active), insoluble à cette concentration.

Ces résultats mettent à nouveau en évidence les particularités de l'espèce $S$. thermophilus du triple point de vue physiologique, nutritionnel et métabolique, ainsi que certaines variations entre souches.

\section{S u m m a ry}

PROPERTIES OF FRUCTOSE 1,6-DIPHOSPHATE INDEPENDENT LACTATE DEHYDROGENASES FROM STEPTOCOCCUS THERMOPHILUS

The properties of $S$. thermophilus LDH have been studied using soluble crude extracts of 10 strains and also partially purified extracts 
of 2 of them. The stability of LDH when refrigerated and frozen as well as its thermoresistance varied with the strains. Phosphate activated LDH but FDP did not ; on the contrary FDP inhibited the LDH activity at concentration $>1 \mathrm{mM}$. Slight inhibition of $\mathrm{LDH}$ occurred with $\mathrm{Mn}^{++}$. The ADP, ATP, 6-P-gluconate, oxamate and NAD were the strongest inhibitors of $\mathrm{LDH}$ activity measured with pyruvate as the substrate. Some of the inhibitions varied with the $\mathrm{pH}$. The activity with $\mathrm{L}(+)$-lactate as the substrate was 30 times lower than the reverse activity (reduction of pyruvate) and lactate concentrations above $200 \mathrm{mM}$ were needed. An excess of NADH (0.2 mM with strain 302; $0.33 \mathrm{mM}$ with strain 385 ) inhibited the LDH activity. Likewise, pyruvate $(>10 \mathrm{mM}$ ) was inhibitory but its effect was different according to the presence or absence of FDP $(0.5 \mathrm{mM})$. The $K_{M}$ and $V_{\max }$ constants were measured. The activity of LDH remained unchanged between $\mathrm{pH} 4.7$ and 7.5 . However this activity rapidly decreased at $\mathrm{pH}<4.7$, becoming almost nil at $\mathrm{pH}$ 4.6. The $S$. thermophilus LDH electrophoretic mobilities were compared to those of the well known isoenzymes of animal origin. Two types of $\mathrm{LDH}$ with different mobilities were found: the A type, soluble in 55 p. 100 ammonium sulphate solution, and the B type (the most active) insoluble at this concentration.

These results confirm the physiological, nutritional and metabolic particularities of $S$. thermophilus species and also the variations found among the strains within the species.

\section{Zusammenfassung}

\section{EIGENSCHAFTEN DER VON FDP-UNABHÄNGIGEN \\ LAKTATDEHYDROGENASEN VON STREPTOCOCCUS THERMOPHILUS}

Untersucht wurden die Eigenschaften der Laktatdehydrogenasen (LDH) von Streptococcus thermophilus. Bei dieser Untersuchung wurden lösliche Rohextrakte von 10 Stämmen und teilweise gereinigte Extrakte von 2 dieser 10 Stämme verwendet.

Die Kälte-, Gefrier- und auch die Wärmebeständigkeit ist unterschiedlich bei den einzelnen Stämmen. Die LDH werden wenig durch das FDP aktiviert oder aber bei starker Konzentration inhibiert ( $>1 \mathrm{mM}$ ). Sie werden durch das Ion $\mathrm{Mn}^{++}$leicht inhibiert, ADP, ATP, 6-P-Gluconat, Oxamat und die NAD sind die stärksten Inhibitoren der LDH-Aktivität bei Pyruvat als Substrat. Einige dieser Inhibitionen hängen vom $\mathrm{pH}$ ab. Die Aktivität bei Oxidation des $\mathrm{L}(+)$-Laktats ist $30 \mathrm{mal}$ schwächer als die umgekehrte Aktivität (Pyruvatreduktion) und erfordert sehr starke Laktatkonzentrationen $(>200 \mathrm{mM})$. Ein UEberschuß an NADH $(0,2 \mathrm{mM}$ bei Stamm 302, $0,33 \mathrm{mM}$ bei Stamm 385) inhibiert die LDH-Aktivität. Das Pyruvat wirkt auch inhibierend ( $>10 \mathrm{mM}$ ) und seine Wirkung hängt davon $\mathrm{ab}$, ob FDP vorhanden ist $(0,5 \mathrm{mM})$ oder nicht. Die $\mathrm{K}_{\mathrm{s}}$ und die $\mathrm{V}_{\max }$ wurden gemessen. Die LDH- 
Aktivität bleibt in einem großen pH-Bereich konstant $(4,7$ bis 7,5$)$. Sie fällt jedoch bei $\mathrm{pH}$-Werten $<4,7$ steil ab und ist bei $\mathrm{pH} 4,6$ so gut wie nicht mehr vorhanden. Die elektrophoretischen Mobilitäten der LDH von $S$. thermophilus wurden mit den sehr gut bekannten Isoenzymen des Rindermuskels verglichen. Es wurden zwei LDH mit verschiedenen Mobilitäten gefunden: Typ A - löslich in 55 p. 100 Ammoniumsulfatlösung und Typ B (der bedeutendste) unlöslich bei dieser Konzentration.

Diese Ergebnisse bestätigen erneut die physiologischen, nutritionellen und metabolischen Eigenschaften der Art S. thermophilus sowie gewisse Schwankungen von einem Stamm zum andern.

Reçu pour publication en septembre 1980.

\section{Bibliographie}

[1] Accolas (J. P.), Bloquel (R.), Didienne (R.) et Régnier (J.) (1977). - Propriétés acidifiantes des bactéries lactiques thermophiles en relation avec la fabrication du yoghourt. Le Lait, 57, 1-23.

[2] Accolas (J. P.), Hemme (D.), Desmazeaud (M. J.), Vassal (L.), Bouillanne (C.) et Veaux (Monique) (1980). - Les levains thermophiles. Propriétés et comportement en technologie laitière. Le Lait, 60, 487-524.

[3] GaRviE (E. I.) (1978). - Lactate dehydrogenases of Streptococcus thermophilus. J. Dairy Res., 45, 515-518.

[4] Garvie (E. I.) (1980). - Bacterial lactate dehydrogenases. Microbiol. Rev., 44, 106-139.

[5] Gordon (G.) and Doelle (H.) (1976). - Purification, properties and immunological relationship of $\mathrm{L}(+)$-lactate dehydrogenase from Lactobacillus casei. Eur. J. Biochem., 67, 543-555.

[6] Hemme (D.) et Nardi (Michèle) (1980). - Electrophorégrammes des protéines, de la $\beta$-galactosidase et de l'aldolase chez Streptococcus thermophilus et les lactobacilles thermophiles. Leurs variations selon les souches et les conditions de culture. Le Lait, 60, 375-392.

[7] Hemme (D.), Nardi (Michèle) et JetTe (D.) (1980). - $\beta$-galactosidases et phospho- $\beta$-galactosidases de Streptococcus thermophilus. Le Lait, 60, 595-618.

[8] Hemme (D.), Wahl (Danièle) et Nardi (Michèle) (1980). - Variations de l'équipement enzymatique de Streptococcus thermophilus. Le Lait, 60, $111-129$.

[9] Hensel (R.), Mayr (U.), Stetter (K. O.) and Kandler (O.) (1977). - Comparative studies of lactic acid dehydrogenases in lactic acid bacteria. I. Purification and kinetics of the allosteric L-lactic dehydrogenase from Lactobacillus casei ssp. casei and Lactobacillus curvatus. Arch. Microbiol., 112, 81-93.

[10] Jonas (H. A.), ANDERS (R. F.) and JAGo (G. R.) (1972). - Factors affecting the activity of the lactate dehydrogenase of Streptococcus cremoris. J. Bacteriol., 111, 397-403.

[11] Lineweaver (H.) and Burk (D.) (1934). - Determination of enzyme dissociation constants. J. Amer. Chem. Soc., 56, 658-666. 
[12] Lowry (O. H.), Rosebrough (N. J.), FarR (A. L.) and Randall (R. J.) (1951). Protein measurement with the Folin phenol reagent. J. Biol. Chem., 193, 265-275.

[13] Mou (L.), Mulvena (D. P.), Jonas (H. A.) and Jago (G. R.) (1972). - Purification and properties of nicotinamide adenine dinucleotide-dependent Dand L-lactate dehydrogenases in group $\mathrm{N}$ Streptococcus. J. Bacteriol., $111,392-396$.

[14] Terzaghi (B.) and Sandine (W. E.) (1975). - Improved medium for lactic streptococci and their bacteriophages. Appl. Microbiol., 27, 807-813.

[15] Wittenberger (C. L.) and Angelo (N.) (1970). - Purification and properties of a fructose-1,6-diphosphate-activated lactate dehydrogenase from Streptococcus faecalis. J. Bacteriol., 101, 717-724.

[16] Wolin (M. J.) (1964). - Fructose 1,6-diphosphate requirement of streptococcal lactic dehydrogenases. Science, 146, 775-777.

[17] Yamada (T.), Endo (K.) and Araya (S.) (1976). - A fructose 1,6-diphosphate independent L-lactate dehydrogenase in a strain of Streptococcus mutans. Arch. Oral Biol., 21, 233-236. 